\title{
AUTOMORPHISMS OF SPACES WITH FINITE FUNDAMENTAL GROUP
}

\author{
GEORGIA TRIANTAFILLOU
}

\begin{abstract}
Let $X$ be a finite $\mathrm{CW}$-complex with finite fundamental group. We show that the group aut $(X)$ of homotopy classes of self-homotopy equivalences of $X$ is commensurable to an arithmetic group. If in addition $X$ is an oriented manifold then the subgroup $\operatorname{aut}_{t}(X)$ of homotopy classes of tangential homotopy equivalences is commensurable to an arithmetic group. Moreover if $X$ is a smooth manifold of dimension $\geq 5$ then the subgroup $\operatorname{diff}(X)$ of $\operatorname{aut}(X)$ the elements of which are represented by diffeomorphisms is also commensurable to an arithmetic group.
\end{abstract}

\section{INTRODUCTION}

Let $X$ be a CW-complex with finite fundamental group. We further assume that $X$ is either a finite $\mathrm{CW}$-complex or a complex whose homotopy groups are finitely generated and vanish beyond a certain dimension. In this note we study homotopy classes of automorphisms of such spaces $X$ which preserve various kinds of additional structures on $X$. We show that certain groups of homotopy classes of automorphisms are commensurable to arithmetic groups.

Arithmetic groups have nice properties. By a result of Borel and HarishChandra $[\mathrm{BH}]$ arithmetic groups are finitely presented. In fact arithmetic groups are groups of finite type by a result of Borel and Serre [BS]. By definition a group $G$ is of finite type if its classifying space $B G$ is homotopy equivalent to a CWcomplex with finitely many cells in each dimension. Being of finite type for a group implies and in fact is much stronger than finite presentation. Two groups are said to be commensurable if there is a finite sequence of homomorphisms between them which have finite kernels and images of finite index.

Theorem 1. The group aut $(X)$ of homotopy classes of self-homotopy equivalences of a finite $C W$-complex $X$ with finite fundamental group is commensurable to an arithmetic group.

The above result is also true if $X$ has finite fundamental group and finitely generated higher homotopy groups which vanish beyond a certain dimension. Theorem 1 generalizes a result of [Wi] for the simply connected case and independently of $[\mathrm{S}]$ for the nilpotent case. It has been shown by [DDK] that

Received by the editors August 25, 1994.

1991 Mathematics Subject Classification. Primary 55P10, 55P62.

Key words and phrases. Homotopy equivalence, diffeomorphism, arithmetic group, minimal model.

(C) 1995 American Mathematical Society 
$\operatorname{aut}(X)$ is of finite type for virtually nilpotent spaces including spaces with finite fundamental group. The full strength of arithmeticity of aut $(X)$ above is needed to prove the following result. We note here that the properties of being of finite type, finitely presented, finitely generated or arithmetic are not in general inherited by subgroups.

Let $M$ be a smooth oriented manifold with finite fundamental group and let

$$
\operatorname{aut}_{t}(M) \subseteq \operatorname{aut}(M)
$$

be the subgroup the elements of which are represented by maps $f$ which preserve the orientation of $M$ and the tangent bundle, i.e.,

$$
f^{*}(T(M))=T(M) \text {. }
$$

These maps are called tangential homotopy equivalences.

Theorem 2. The group aut $(M)$ of homotopy classes of tangential self-homotopy equivalences of an oriented manifold $M$ with finite fundamental group is commensurable to an arithmetic group.

Let $\operatorname{diff}(M)$ be the subgroup of aut $_{t}(M)$ the elements of which are represented by diffeomorphisms.

Theorem 3. Let $M$ be a smooth closed oriented manifold of dimension $\geq 5$ which has finite fundamental group. Then the group of homotopy classes of orientation preserving diffeomorphisms $\operatorname{diff}(M)$ is commensurable to an arithmetic group.

In the proofs of Theorem 1 and 2 we employ minimal model techniques in the equivariant context and certain facts from the theory of algebraic and arithmetic groups. In the presence of a finite fundamental group we work with the minimal model in the sense of Sullivan of the universal cover of $M$, where the model is now equipped with an action of $\pi_{1}(X)$.

In the proof of Theorem 3 we apply surgery techniques and the Atiyah-Singer $G$-signature theorem to relate the group of tangential homotopy equivalences to the group diff $M$ of homotopy classes of diffeomorphisms. Out of this comparison follows in particular

Theorem 4. Under the same assumptions as in Theorem 3 the group of homotopy classes of tangential simple self-homotopy equivalences $\operatorname{aut}_{t}^{s}(M)$ is commensurable to an arithmetic group.

Remark. The PL and Top versions of Theorem 3 are also true. All arguments carry over to these categories by using the appropriate versions of the surgery exact sequence and the PL and Top versions of the Atiyah-Singer $G$-signature theorem [W] Theorem 13B.1 and Theorem 14B.2 respectively.

An announcement of the results of this paper appeared in the Bulletin of the American Mathematical Society [T3].

This research was partially supported by a summer research grant from Temple University. I would also like to thank the University of Chicago for its hospitality while part of this work was done.

\section{TANGENTIAL HOMOTOPY EQUIVALENCES}

Let $X$ be a CW-complex with finite $\pi_{1}(X)$ which we denote with $\pi$ for brevity. We consider the universal cover $\tilde{X}$ with the action of $\pi$. Given any 
simply connected CW-complex $Y$ let $A(Y)$ be the piecewise linear de Rham complex of polynomial forms over $\mathbf{Q}$ as in [S]. We recall that the minimal model $\mathscr{M}$ of $Y$ is a free minimal differential graded commutative algebra over $\mathbf{Q}$ which can be mapped into $A(Y)$ via a cohomology isomorphism

$$
\rho: \mathscr{M} \rightarrow A(Y) \text {. }
$$

The minimal model has a natural filtration of subalgebras

$$
\mathbf{Q} \subseteq \ldots \mathscr{M}(n) \subseteq \mathscr{M}(n+1) \ldots \subseteq \mathscr{M},
$$

where $\mathscr{M}(n)$ is the subalgebra generated by elements of degree less or equal to $n$. The subalgebra $\mathscr{M}(n+1)$ is constructed from $\mathscr{M}(n)$ by adjoining generators of degree $n+1$, i.e., $\mathscr{M}(n+1)$ is the tensor product of $\mathscr{M}(n)$ with an exterior or polynomial algebra generated by a vector space $V_{n+1}$ of degree $n+1$ depending on the parity of $n+1$.

If the space $Y$ is equipped with the action of a finite group $\pi$ then the minimal model $\mathscr{M}$ can be provided with an action of $\pi$ and the map $\rho$ can be constructed to be $\pi$-equivariant. Moreover this $\pi$-minimal model is unique up to equivariant isomorphism [T1]. This is due to the fact that the group ring $\mathbf{Q}(\pi)$ is semisimple when $\pi$ is finite and therefore all lifting and splitting properties of the usual linear maps of vector spaces over $\mathbf{Q}$ carry over to the $\pi$-equivariant case. In the inductive construction of $\mathscr{M}$ the subalgebras $\mathscr{M}(n)$ and the vector spaces $V_{n+1}$ can be chosen to have a $\pi$-action.

The $\pi$-minimal model provides the connection with algebraic groups as stated in the following theorem. First we give the notion of homotopy in this algebraic context. Let $f, g: \mathscr{A} \rightarrow \mathscr{B}$ be two equivariant maps between two $\pi$-dga's and let $\Lambda(t, d t)$ be the free acyclic dga generated by the element $t$ of degree 0 and its differential $d t$.

Definition 5. The maps $f$ and $g$ are equivariantly homotopic if there is a $\pi$ equivariant map

$$
F: \mathscr{A} \rightarrow \mathscr{B} \otimes \Lambda(t, d t)
$$

such that the projections for $t=0$ and $t=1$ are equal to $f$ and $g$ respectively.

It is shown in [T1] that $\pi$-homotopy has the same lifting and extension properties as the usual homotopy for dga's. In particular it is an equivalence relation if $\mathscr{A}$ is a free dga.

Let $\operatorname{Aut}(\mathscr{M})$ be the group of automorphisms of $\mathscr{M}$ and let $\operatorname{Aut}(\mathscr{M})^{\pi}$ be the subgroup of $\pi$-equivariant automorphisms. We denote with aut $(\mathscr{M})_{\pi}$ the group of $\pi$-homotopy classes of $\pi$-automorphisms of $\mathscr{M}$. We will show the following:

Theorem 6. (i) The group aut $(\mathscr{M})_{\pi}$ is an algebraic matrix group over $\mathbf{Q}$.

(ii) The group aut $(\mathscr{M})_{\pi}$ acts algebraically on the equivariant cohomology of $\mathscr{M}$ with any coefficients.

The case when $\pi$ is trivial is due to Sullivan [S]. The equivariant case follows along similar lines. Before we give the proof we recall some definitions and facts about algebraic and arithmetic groups from [BH].

Let $V$ be an $n$-dimensional rational vector space and let $G L(V)$ denote the group of linear automorphisms of $V$. Consider the elements of $G L(V)$ as matrices with entries in $\mathbf{Q}$. 
Definition 7. A group $K \subseteq G L(V)$ is called a linear algebraic group over $\mathbf{Q}$ or an algebraic matrix group over $\mathbf{Q}$ if it is the annihilator of a set of polynomials on the entries with rational coefficients.

Definition 8. A group homomorphism $f: G \rightarrow G^{\prime}$ between two linear algebraic groups is said to be algebraic if the entries of $f(g)$ are polynomial functions of the entries of $g \in G$.

Definition 9. Let $L$ be a lattice in $V$, i.e., a finitely generated subgroup of maximal rank and let $K_{L}$ be the subgroup of elements $g \in K$ such that $g(L)=$ $L$. Then $K_{L}$ is said to be an arithmetic subgroup of $K$.

A different choice of lattice $L^{\prime}$ leads to a commensurable subgroup $K_{L^{\prime}}$, i.e., $K_{L} \cap K_{L^{\prime}}$ is a subgroup of finite index in both $K_{L}$ and $K_{L^{\prime}}$. Thus the commensurability class of the arithmetic subgroups of an algebraic group is an invariant of the algebraic group.

The proof of Theorem 6 is based on the following proposition.

Proposition 10. Let $\mathscr{M}$ be a minimal simply connected algebra with an action of a finite group $\pi$ and let $f: \mathscr{M} \rightarrow \mathscr{M}$ be an equivariant isomorphism. Then $f$ is equivariantly $\pi$-homotopic to the identity if and only if there is an equivariant derivation $i: \mathscr{M} \rightarrow \mathscr{M}$ of degree -1 such that

$$
f=\exp (d i+i d)=I+(d i+i d)+(1 / 2)(d i+i d)^{2}+\ldots .
$$

Moreover if $H$ is a homotopy between $f$ and the identity $e$, then $i$ can be chosen in such a fashion that the homotopy $H^{\prime}$ induced by $i$ and the given homotopy $H$ are homotopic by a homotopy $F$ such that $\left.F\right|_{t=1}=f$ and $\left.F\right|_{t=0}=$ $e$.

Remark. The existing proof in the literature [H] of the existence of $i$ for the nonequivariant case is rather roundabout as it involves passing to an alternative definition of homotopy of maps of dga's. Although it can be easily adapted to the equivariant case we will follow here a different approach which keeps track of the way $i$ depends on a given homotopy between $f$ and the identity. The explicit construction of $F$ along with its boundary conditions turns out to be advantageous in many cases where one notion of homotopy works better than the other. This will become apparent in a subsequent paper of ours where we study the group of isotopy classes of diffeomorphisms of a manifold, and where the constructions on the minimal model level have to reflect geometric constructions which are more refined than homotopy constructions. Thus Proposition 10 for trivial $\pi$ complements the literature for the simply connected case as well.

Proof. First we recall from the nonequivariant case that if $i$ exists and has the stated properties then $i$ induces a homotopy between $f$ and $e$ as follows. Let

$$
i^{\prime}: \mathscr{M} \otimes \mathbf{Q}(t, d t) \rightarrow \mathscr{M} \otimes \mathbf{Q}(t, d t)
$$

be the induced derivation, where $i^{\prime}(x)=t i(x)$ on generators of $\mathscr{M}$ and $i^{\prime}(t)=$ 0 . Then

$$
H^{\prime} \equiv l \circ \exp \left(d i^{\prime}+i^{\prime} d\right)
$$

is the induced homotopy, where $l: \mathscr{M} \rightarrow \mathscr{M} \otimes \mathbf{Q}(t, d t)$ is the inclusion. This makes perfect sense equivariantly. 
Let $H: \mathscr{M} \rightarrow \mathscr{M} \otimes \mathbf{Q}(t, d t)$ be a homotopy between a map $f$ and the identity which we denote $e$. Since $f$ is nilpotent the expression

$$
D \equiv \sum(-1)^{n-1}(1 / n)(f-e)^{n}
$$

makes sense and therefore $f$ can be written $f=\exp (D)$. In order to find $i$ such that $D=d i+i d$ we proceed inductively over the minimal subalgebras $\mathscr{M}(n)$. On $\mathscr{M}(2)$, which consists entirely of cocycles, $D x=0$.

Assume inductively that $i$ is defined on $\mathscr{M}(n)$ such that the homotopies $\left.H\right|_{\mathscr{M}(n)}$ and $H^{\prime}=l \circ \exp \left(d i^{\prime}+i^{\prime} d\right)$ are homotopic by a homotopy

$$
F: \mathscr{M} \rightarrow \mathscr{M} \otimes \mathbf{Q}(t, d t, s, d s) .
$$

Moreover assume that

$$
\left.F\right|_{t=1}=f \text { and }\left.F\right|_{t=0}=e
$$

i.e., they are both independent of the variable $s$.

Now define $i(x)$ on generators of degree $n+1$ by the formula

$$
\begin{aligned}
i(x)= & -A-\int_{0}^{1}\left(i^{\prime} d(x)+(1 / 2 !)\left(i^{\prime} d\right)^{2}(x)+\ldots+(1 / n !)\left(i^{\prime} d\right)^{k}\right) \\
& +\int_{0}^{1} \int_{0}^{1} F(d x)+\int_{0}^{1} H(x) .
\end{aligned}
$$

Here $A$ is the unique element defined by the formula

$$
d A=\left(i d(x)+(1 / 2 !)(d i+i d)^{2}(x)+\ldots\right)-\left(i d(x)+(1 / 2 !)(i d)^{2}(x)+\ldots\right),
$$

where all terms which are not coboundaries are canceled by opposite signs. Averaging $i$ on the generators by the action of $\pi$ and extending it by the product formula provides an equivariant map. Also define

$$
i^{\prime}(x)=t i(x)
$$

and

$$
H^{\prime}(x)=\exp \left(d i^{\prime}+i^{\prime} d\right)(x) \text {. }
$$

We recall that the operation $\int_{0}^{t}$ on $\mathscr{M} \otimes \mathbf{Q}(t, d t)$ is defined by $\int_{0}^{t} a \otimes t^{k}=0$ and

$$
\int_{0}^{t} a \otimes t^{k} d t=(-1)^{\operatorname{deg} a} a \otimes \frac{t^{k+1}}{k+1},
$$

for $a \in \mathscr{M}$. It satisfies the basic formula

$$
\left.b\right|_{t=1}-\left.b\right|_{t=0}=d \int_{0}^{1} b+\int_{0}^{1} d b
$$

for $b \in \mathscr{M} \otimes \mathbf{Q}(t, d t)$. It is a straightforward but tedious exercise to check that $f(x)=\exp (d i+i d)$, and $H^{\prime}(d x)=d H^{\prime}(x)$. Here one uses the formulas

$$
f(x)-x=\int_{0}^{1} H(d x)+d \int_{0}^{1} H(x)
$$

and, similarly,

$$
\begin{aligned}
& \int_{0}^{1} H^{\prime}(d x)-\int_{0}^{1} H(d x)=\int_{0}^{1} d \int_{0}^{1} F(d x) \\
& \quad=-d \int_{0}^{1} \int_{0}^{1} F(d x)+\left.\int_{0}^{1} F(d x)\right|_{t=1}-\left.\int_{0}^{1} F(d x)\right|_{t=0}=-d \int_{0}^{1} \int_{0}^{1} F(d x) .
\end{aligned}
$$


We note that we have the freedom of changing the value $i(x)$ on the new generators of degree $n+1$ by a cocycle since such a change leaves $f(x)=$ $\exp (d i+i d)(x)$ invariant and changes $H^{\prime}(x)$ only by a coboundary. We will choose this cocycle appropriately so that the extension of the homotopy $F$ satisfies the necessary boundary properties. We extend the homotopy $F$ by the usual obstruction theory. Namely the obstruction

$$
\mathscr{O} \equiv H^{\prime}(x)-H(x)-\int_{0}^{1} F(d x)
$$

being a cocycle which projects to 0 for $t=0$ and $t=1$ respectively, is a coboundary, say, $d v$. Moreover $v$ can be chosen to project also to 0 for $t=0$. However there may not be any $v$ satisfying in addition $\left.v\right|_{t=1}=0$. The condition on the obstruction $\mathscr{O}$ for that to happen is

$$
\int_{0}^{1}\left(H^{\prime}(x)-H(x)-\int_{0}^{1} F(d x)\right)=d u
$$

for some $u \in \mathscr{M}$. If this last integral is a cocycle $c$ we have to change $H^{\prime}(x)$ (by changing $i(x))$ in such a way that the integral becomes a coboundary. By computation we get

$$
\int_{0}^{1} H^{\prime}(x)=\left(i(x)+\frac{1}{2}(i d) i(x)+\ldots+\frac{1}{k !}(i d)^{k-1} i(x)\right)+d w
$$

for some $w$ which does not depend on $i(x)$. We see clearly that by changing $i(x)$ to $i(x)-c$, the new $H^{\prime}(x)$ satisfies the required condition

$$
\int_{0}^{1} H^{\prime}(x)-\int_{0}^{1} H(x)-\int_{0}^{1} \int_{0}^{1} F(d x)=d u .
$$

This guarantees that $v$ can be chosen to vanish when projected to $t=0$ and $t=1$. Namely take

$$
v \equiv \int_{0}^{t}\left(H^{\prime}(x)-H(x)-\int_{0}^{1} \cdot F(d x)\right)-d(t u)
$$

Now define

$$
F(x)=H(x)+\int_{0}^{s} F(d x)+d(v \otimes s) .
$$

We can verify $\left.F(x)\right|_{t=1}=f(x)$ and $\left.F(x)\right|_{t=0}=x$ as required by the induction hypothesis. This completes the proof of the proposition.

Proof of Theorem 6. (i) We first observe that the group of automorphisms $\operatorname{Aut}(\mathscr{M})$ is an algebraic group and the group of equivariant automorphisms $\operatorname{Aut}(\mathscr{M})^{\pi}$ is an algebraic subgroup satisfying a finite number of additional linear equations. We denote with $\operatorname{aut}(\mathscr{M})_{\pi}$ the quotient of the latter group with the group $A$ of $\pi$-automorphisms which are equivariantly homotopic to the identity. As in the nonequivariant case [S], the way to show that this quotient is an algebraic group is to show that the subgroup $A$ is the image under exp of a nilpotent Lie algebra. This is done by Proposition 10 and the fact that the equivariant derivations of the form $d i+i d$ form such a Lie algebra.

(ii) In order to show that aut $(\mathscr{M})_{\pi}$ acts algebraically on the cohomology $H^{*}(\mathscr{M}) \equiv H$ we have to show that the map

$$
\operatorname{aut}(\mathscr{M})_{\pi} \rightarrow \operatorname{Aut}(H)^{\pi}
$$


is an algebraic map. It is easy to see that the map

$$
\operatorname{Aut}(\mathscr{M})_{\pi} \rightarrow \operatorname{Aut}(H)^{\pi}
$$

is algebraic by restricting the automorphisms to the subspace of cocycles and then taking quotients. Both operations are algebraic. On the other hand we know that the map

$$
\operatorname{Aut}(\mathscr{M})^{\pi} \rightarrow \operatorname{aut}(\mathscr{M})_{\pi}
$$

is algebraic as we argued above. By a theorem in algebraic groups [Hu] the factorization aut $(\mathscr{M})_{\pi} \rightarrow \operatorname{Aut}(H)^{\pi}$ is also algebraic. This completes the proof of Theorem 6.

In general there are many $\pi-\mathrm{CW}$-complexes which are not $\pi$-homotopy equivalent rationally but have the same $\pi$-minimal model. However when we restrict to free $\pi$-actions on simply connected spaces this correspondence is one-to-one [BSz].

More precisely we fix a map $p: X \rightarrow B \pi$ which induces an isomorphism on $\pi_{1}()$, where $B \pi$ is the classifying space of $\pi$, and we convert $p$ into a fibration $p^{\prime}: X^{\prime} \rightarrow B \pi$ in the usual functorial way. Since there is a canonical homotopy equivalence $i: X \rightarrow X^{\prime}$ such that $p^{\prime} \circ i=p$ we will not distinguish between $X$ and $X^{\prime}$ in the sequel using the fibration or CW-complex properties depending on the context. By abuse of terminology we will also identify $p$ with $p^{\prime}$. Let $\tilde{X}$ be the universal cover of $X$. Similarly as above we can consider $\tilde{X}$ as a $\mathrm{CW}$-complex or as the homotopy equivalent space defined to be the fiber product in the diagram

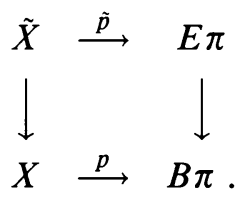

Since $\tilde{X}$ is simply connected one can construct its rationalization $\tilde{X}_{0}$ with a free $\pi$-action in such a fashion that the fibration $\tilde{p}$ factors through a fibration $\tilde{p}_{0}: \tilde{X}_{0} \rightarrow E \pi$. We denote with $X_{0}$ the quotient space $\tilde{X}_{0} / \pi$.

Throughout this paper we follow the convention that Aut denotes actual automorphisms of a space or an algebra whereas aut denotes homotopy classes of such automorphisms. In particular $\operatorname{Aut}(X)$ denotes the monoid of selfhomotopy equivalences of $X$ and $\operatorname{aut}(X)$ the group of homotopy classes of self-homotopy equivalences. The subscript $*$ indicates base point preserving automorphisms.

Let $\operatorname{aut}_{*}(X)_{B \pi}$ be the group of base point preserving homotopy classes of homotopy equivalences of $X$ over $B \pi$, i.e., maps and homotopies which cover the identity on $B \pi$. Similarly let $\operatorname{aut}_{*}(\tilde{X})_{E \pi}$ be the group of equivariant homotopy classes of base point preserving equivariant homotopy equivalences of $\tilde{X}$ over $E \pi$. We observe that these two groups are isomorphic. On the other hand let aut $(\mathscr{M})_{\pi}$ be the group of equivariant homotopy classes of $\pi$-isomorphisms of the $\pi$-minimal model $\mathscr{M}$ of $\tilde{X}$. It can be easily deduced from [BSz] that

$$
\operatorname{aut}_{*}\left(\tilde{X}_{0}\right)_{E \pi} \tilde{=} \operatorname{aut}(\mathscr{M})_{\pi} .
$$

Theorem 11. The group $\operatorname{aut}_{*}(\tilde{X})_{E \pi}$ is commensurable to an arithmetic subgroup of the group aut $(\mathscr{M})_{\pi}$. 
Proof. We consider the Postnikov decomposition of $\tilde{X}$ over $E \pi$, where all maps $\tilde{X}_{n+1} \rightarrow \tilde{X}_{n}$ are equivariant inclusions. As a result the induced maps of the de Rham algebras are surjective and this enables the construction of the minimal model of $\tilde{X}$ as the union of the models of the $\tilde{X}_{n}$ 's via the commutative diagrams

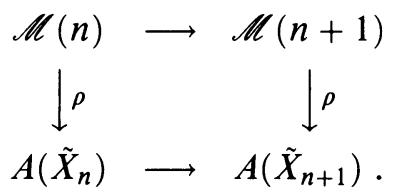

The proof of arithmeticity works exactly as in the nonequivariant case by induction over the Postnikov terms $\tilde{X}_{n}$. We assume inductively that aut ${ }_{*}\left(\tilde{X}_{n}\right)_{E \pi}$ is commensurable to an arithmetic subgroup of aut $(\mathscr{M}(n))_{\pi}$, starting with the fact that $\mathbf{Q}$ with the trivial $\pi$-action is the equivariant minimal model of $A(E \pi)$ if $\pi$ is finite [BSz]. More precisely we assume that for every arithmetic subgroup $\Gamma$ of aut $(\mathscr{M}(n))_{\pi}$ the intersection $\Gamma \cap r\left(\operatorname{aut}_{*}\left(\tilde{X}_{n}\right)_{E \pi}\right)$ has finite index in $\Gamma$ and $r\left(\operatorname{aut}_{*}\left(\tilde{X}_{n}\right)_{E \pi}\right)$ respectively. Here

$$
r: \operatorname{aut}_{*}\left(\tilde{X}_{n}\right)_{E \pi} \rightarrow \operatorname{aut}(\mathscr{M}(n))_{\pi}
$$

is defined as in the nonequivariant case by lifting maps of spaces to maps of the de Rham algebras and then to maps of the minimal models.

We have a commutative diagram of groups

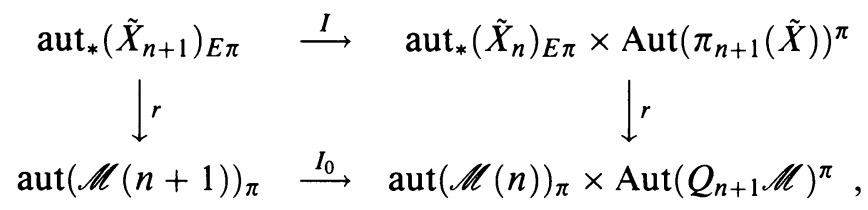

where $Q \mathscr{M}$ is the space of indecomposables of $\mathscr{M}$, i.e., $\mathscr{M}$ is divided out by the subspace of decomposable elements. We note that $I_{0}$ is a map of algebraic groups. We specify the kernel and image of the map $I$ and the map $I_{0}$.

The group aut ${ }_{*}\left(\tilde{X}_{n}\right)_{E \pi} \times \operatorname{Aut}\left(\pi_{n+1}(\tilde{X})\right)^{\pi}$ acts on the equivariant cohomology group $H_{\pi}^{n+2}\left(\tilde{X}_{n} ; \pi_{n+1}(\tilde{X})\right)$ by acting on $\tilde{X}_{n}$ and on the coefficients $\pi_{n+1}(\tilde{X})$, and the image of $I$ coincides with the isotropy group of the $k$-invariant. Similarly $\operatorname{im}\left(I_{0}\right)$ is the isotropy group of the rational $k$-invariant under the action of aut $(\mathscr{M}(n))_{\pi} \times \operatorname{Aut}\left(Q_{n+1}(\mathscr{M})\right)^{\pi}$ on $H_{\pi}^{n+2}\left(\mathscr{M}(n) ; Q_{n+1}\right)$. By Theorem 6 this action is algebraic and therefore the isotropy group $\operatorname{im}\left(I_{0}\right)$ is an algebraic subgroup of aut $(\mathscr{M}(n))_{\pi} \times \operatorname{Aut}\left(Q_{n+1}(\mathscr{M})\right)^{\pi}$. Moreover $\operatorname{im}(I)$ is commensurable to an arithmetic subgroup of $\operatorname{im}\left(I_{0}\right)$ (cf. [B], p. 97).

The kernel of $I$ can be computed by obstruction theory. Let $p r_{1}$ denote the projection into the first factor. First we recall that the kernel of the map

$$
p r_{1} \circ I: \operatorname{aut}_{*}\left(\tilde{X}_{n+1}\right)_{E \pi} \rightarrow \operatorname{aut}_{*}\left(\tilde{X}_{n}\right)_{E \pi}
$$

is an orbit of the action of $H_{\pi}^{n+1}\left(\tilde{X}_{n+1} ; \pi_{n+1}(\tilde{X})\right)$ on $\operatorname{aut}\left(\tilde{X}_{n+1}\right)$, i.e., it is a quotient of $H_{\pi}^{n+1}\left(\tilde{X}_{n+1} ; \pi_{n+1}(\tilde{X})\right)$.

The additional condition that the elements of $\operatorname{ker} I$ induce the identity on $\pi_{n+1}(\tilde{X})$ identifies $\operatorname{ker} I$ as a quotient of the kernel of the map

$$
\delta: H_{\pi}^{n+1}\left(\tilde{X}_{n+1} ; \pi_{n+1}\right) \rightarrow H_{\pi}^{n+2}\left(\tilde{X}_{n}, \tilde{X}_{n+1} ; \pi_{n+1}\right) .
$$


We observe here that the latter group is just $\operatorname{Hom}\left(\pi_{n+1}(\tilde{X})\right)^{\pi}$. By the long exact cohomology sequence the kernel of $\delta$ is $H_{\pi}^{n+1}\left(\tilde{X}_{n} ; \pi_{n+1}(\tilde{X})\right)$. We conclude that $\operatorname{ker} I$ is a quotient of $H_{\pi}^{n+1}\left(\tilde{X}_{n} ; \pi_{n+1}(\tilde{X})\right)$. Moreover one can verify by using obstruction theory that $\operatorname{ker} I$ is an abelian subgroup of $\operatorname{aut}_{*}\left(\tilde{X}_{n+1}\right)_{E \pi}$ and that this group structure coincides with the group structure of the above cohomology group. Similarly $\operatorname{ker} I_{0}$ can be identified with a quotient of $H_{\pi}^{n+1}\left(\mathscr{M}(n) ; Q_{n+1}\right)$ and $\operatorname{ker} I$ is commensurable with a lattice in $\operatorname{ker} I_{0}$, i.e., with a finitely generated subgroup of $\operatorname{ker} I_{0}$ of maximal rank.

We consider the following commutative diagram of short exact sequences, where the lower sequence is a sequence of algebraic groups and algebraic maps.

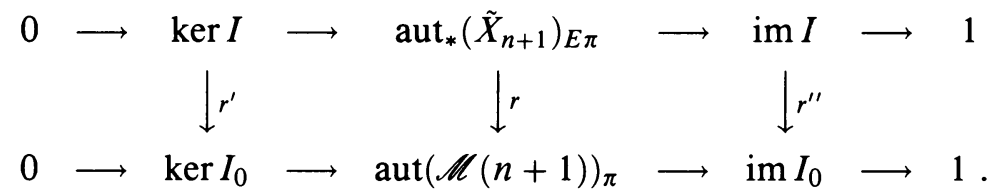

It is obvious that the middle vertical map $r$ has finite kernel since the kernels of the left- and right-hand side maps $r^{\prime}$ and $r^{\prime \prime}$ are torsion.

Now let $\Gamma_{i}$ be any arithmetic subgroup of aut $(\mathscr{M}(i))_{\pi}$ for $i=n$ or $i=n+1$. Then the group $\Gamma^{\prime} \equiv r^{\prime}(\operatorname{ker} I) \cap \Gamma_{n+1}$ is an arithmetic subgroup of $\operatorname{ker} I_{0}$, i.e., it is a lattice in the vector space $\operatorname{ker} I_{0}$. Let $\Gamma_{(k)}$ be the isotropy group of the $k$ invariant under the action of $\Gamma_{n} \times \operatorname{Aut}\left(\pi_{n+1}\right)^{\pi}$ on the cohomology above. Since the action is algebraic the isotropy group is arithmetic. The map

$$
I_{0}: \operatorname{aut}(\mathscr{M}(n+1))_{\pi} \rightarrow \operatorname{im} I_{0}
$$

is a surjective algebraic map and therefore the image $I_{0}\left(\Gamma_{n+1}\right)$ in $\operatorname{im} I_{0}$ is commensurable to $\Gamma_{(k)}[\mathrm{BH}]$.

Denote $\Gamma^{\prime \prime} \equiv I_{0}\left(\Gamma_{n+1}\right) \cap \Gamma_{(k)}$ and $\Gamma \equiv\left(I_{0} \mid \Gamma_{n+1}\right)^{-1}\left(\Gamma^{\prime \prime}\right)$. We have a short exact sequence of groups

$$
0 \rightarrow \Gamma^{\prime} \rightarrow \Gamma \rightarrow \Gamma^{\prime \prime} \rightarrow 1,
$$

each of which is a subgroup of finite index of an arithmetic subgroup in the corresponding algebraic group in the diagram above. Now the subgroups $r^{\prime \prime}(\mathrm{im} I)$ and $\Gamma^{\prime \prime}$ of $\operatorname{im} I_{0}$ are commensurable since by the inductive assumption aut $_{*}\left(\tilde{X}_{n}\right)_{E \pi}$ and $\Gamma_{n}$ and therefore $\operatorname{im} I$ and $\Gamma_{(k)}$ are commensurable. By a straightforward diagram chase involving the three exact sequences above we can show that aut ${ }_{*}\left(\tilde{X}_{n+1}\right)_{E \pi}$ and $\Gamma$ and therefore aut $\left(\tilde{X}_{n+1}\right)_{E \pi}$ and $\Gamma_{n+1}$ are commensurable. This completes the inductive step of the proof.

For finite complexes $X$ we observe that

$$
\operatorname{aut}_{*}(\tilde{X})_{E \pi}=\operatorname{aut}_{*}\left(\tilde{X}_{n}\right)_{E \pi}
$$

for $n$ sufficiently large.

A similar explicit argument has been given in [T2] for the equivariant case when the group action has fixed points.

Proposition 12. The group aut $(X)$ is commensurable to $\operatorname{aut}_{*}(\tilde{X})_{E \pi}$.

Proof. First we note that $\operatorname{aut}(X)$ is commensurable to $\operatorname{aut}_{*}(X)$ of homotopy classes of base point preserving self-homotopy equivalences as can be seen from the fibration

$$
\operatorname{Aut}_{*}(X) \rightarrow \operatorname{Aut}(X) \rightarrow X
$$


and the long exact sequence of homotopy. In particular looking at the $\pi_{0}$ level, we get that

$$
\operatorname{aut}_{*}(X) \rightarrow \operatorname{aut}(X)
$$

is surjective and it has finite kernel. Next we observe that $\operatorname{aut}_{*}(X)$ is commensurable to its subgroup aut $\operatorname{au}_{*}(X)_{\pi}$, where the latter contains the maps which induce the identity on $\pi_{1}(X)=\pi$, because their quotient is a subgroup of $\operatorname{Aut}(\pi)$ and therefore it is finite.

The group aut ${ }_{*}(X)_{\pi}$ can be considered as the group of homotopy classes of self-homotopy equivalences which cover the map

$$
p: X \rightarrow B \pi
$$

up to homotopy, whereas aut ${ }_{*}(X)_{B \pi}$ is the group of homotopy classes of selfhomotopy equivalences which are actual lifts of $p: X \rightarrow B \pi$. The forgetful map

$$
\operatorname{aut}_{*}(X)_{B \pi} \rightarrow \operatorname{aut}_{*}(X)_{\pi}
$$

is surjective and the kernel is a quotient of $[X, \pi]$ and therefore it is finite. This can be easily seen by a Barratt-Puppe sequence type argument. On the other hand

$$
\operatorname{aut}_{*}(X)_{B \pi}=\operatorname{aut}_{*}(\tilde{X})_{E \pi} .
$$

This completes the proof of the proposition.

Corollary 13. The group aut $(X)$ is commensurable to an arithmetic group.

Remark. Because of Proposition 12 we will neglect base point considerations in the sequel.

Theorem 14. The group of tangential self homotopy equivalences aut ${ }_{t}(X)$ is commensurable to an arithmetic group.

Proof. Let $t \in[X, B S O]$ be the homotopy class of the classifying map of the tangent bundle. The group aut $\operatorname{au}_{t}(X)$ is the isotropy group of $t$ under the action of $\operatorname{aut}(X)$ on $[X, B S O]$ by composition. By rationalizing we have the action of $\operatorname{aut}(\mathscr{M})_{\pi}$ on $H^{4 *}(X ; \mathbf{Q})$ which is algebraic by Theorem 6 . Let

$$
p \in\left[X, B S O_{0}\right]=H^{4 *}(X ; \mathbf{Q})
$$

be the Pontryagin class, i.e., the image of $t$ under rationalization. The isotropy group

$$
K \subseteq \operatorname{aut}(\mathscr{M})_{\pi}
$$

of $p$ is an algebraic subgroup, and the intersection of $K$ with the image of aut $(X)$ is an arithmetic subgroup $A \subset K[\mathrm{Hu}]$. Now aut ${ }_{t}(X)$ is commensurable to $A$ as can be easily seen from the fact that

$$
[X, B S O] \rightarrow\left[X, B S O_{0}\right]
$$

is a finite-to-one map. The latter fact can be easily established by an inductive argument over the Postnikov tower of $B S O$. This completes the proof.

\section{HOMOTOPY CLASSES OF DIFFEOMORPHISMS}

Let $M$ be a smooth closed orientable manifold of dimension $\geq 5$ which has finite fundamental group. Let $\operatorname{diff}(M)$ be the subgroup of $\operatorname{aut}(M)$ consisting of the classes which can be represented by orientation preserving diffeomorphisms. Then $\operatorname{diff}(M) \subseteq \operatorname{aut}_{t}(M)$. We show the following: 
Proposition 15. The group $\operatorname{diff}(M)$ is a subgroup of finite index in aut $_{t}(M)$.

Corollary 16. The group $\operatorname{diff}(M)$ is commensurable to an arithmetic group.

Proof of Proposition 15. We consider the surgery exact sequence

$$
\ldots L_{n+1}(\pi) \rightarrow \mathscr{S}(M) \rightarrow \mathscr{T}(M) \rightarrow L_{n}(\pi)
$$

as in [W]. In fact we consider the homotopy rather than the simple hmotopy version of it.

The structure set $\mathscr{S}(M)$ consists of equivalence classes of pairs $(N, f)$ where $N$ is an oriented smooth manifold and $f: N \rightarrow M$ is an orientation preserving homotopy equivalence. Two pairs $(N, f)$ and $\left(N^{\prime}, f^{\prime}\right)$ are equivalent if there is a diffeomorphism $\alpha: N \rightarrow N^{\prime}$ such that $f$ is homotopic to $f^{\prime} \alpha$. We identify the elements of $\mathscr{T}(M)$ with equivalence classes of pairs $(v, h)$, where $v$ is a vector bundle, and $h$ is a fibre homotopy equivalence between $v$ and the tangent bundle $\tau$. Two pairs $(v, h)$ and $\left(v^{\prime}, h^{\prime}\right)$ are equivalent if there is a vector bundle $V$ over $M \times I$ and a fiber homotopy equivalence $H$ from $V$ to $\tau$ such that $\left.H\right|_{M \times 0}=h$ and $\left.H\right|_{M \times 1}=h^{\prime}$. The identity element is $(\tau$, id) .

We consider the action of aut $(M)$ on $\mathscr{S}(M)$ and $\mathscr{T}(M)$ by composition of maps and pulling back bundles. Let $\operatorname{aut}_{\tau}(M)$ be the isotropy group of the identity element of $\mathscr{T}(M)$ under the action of aut $(M)$. Similarly aut $t_{t}(M)$ can be considered as the isotropy group of the tangent bundle $[t] \in[M, B O]$ under the action of aut $(M)$. The forgetful map

$$
F: \mathscr{T}(M) \rightarrow[M, B O]
$$

is equivariant with respect to this action and hence

$$
\operatorname{aut}_{\tau}(M) \subseteq \operatorname{aut}_{t}(M) .
$$

We recall that we can naturally identify

$$
\mathscr{T}(M)=[M, G / O]
$$

Since the fiber $[M, G]$ of the forgetful map $[M, G / O] \rightarrow[M, B O]$ is finite the two groups $\operatorname{aut}_{\tau}(M)$ and $\operatorname{aut}_{t}(M)$ are commensurable. Therefore, in order to prove the proposition it suffices to show that $\operatorname{diff}(M)$ is a subgroup of finite index of $\operatorname{aut}_{\tau}(M)$.

We consider the action of aut $\tau_{\tau}(M)$ on the structure set $\mathscr{S}(M)$. The isotropy group of the element [id] $\equiv[M$, id $] \in \mathscr{S}(M)$ is $\operatorname{diff}(M)$, and its orbit $\mathscr{O}$ under the action of $\operatorname{aut}_{\tau}(M)$ is isomorphic to the quotient

$$
\mathscr{O}=\operatorname{aut}_{\tau}(M) / \operatorname{diff}(M) \text {. }
$$

We will show that this orbit is a finite set.

First we observe that this orbit is a subset of the kernel of the map $\mathscr{S}(M) \rightarrow$ $[M, G / O]$. Let $[f]$ be an element of the orbit, where $f: M \rightarrow M$ is a selfhomotopy equivalence with the required properties. Then $[f]$ is in the orbit $\mathscr{O}^{\prime}$ of [id] under the action of $L_{n+1}(\pi)$ as well. That is

$$
[f]=l[\mathrm{id}],
$$

where $l$ is the obstruction to a homotopy equivalence of a cobordism $F: W \rightarrow$ $M \times I$ such that

$$
\partial W=M \cup(-M)
$$


and $F$ coincides with id and $f$ on the two ends.

If $n+1$ is odd then $L_{n+1}(\pi)$ is finite and we are done.

If $n+1=2 k$ then we argue as follows. We consider the multisignature

$$
\chi: L_{2 k}(\pi) \rightarrow \mathbf{R}(\pi)
$$

as in [W], p. 167 or [P]. This map has finite kernel. It follows easily from the definitions that the image $\chi(l)$ is equal to the $\pi$-signature $\sigma(\tilde{W}) \in \mathbf{R}(\pi)$ of the universal cover $\tilde{W}$ of $W$ in the sense of Atiyah and Singer [AS].

Now consider the universal cover $\tilde{M}$ of $M$ which is an odd dimensional closed manifold with a free $\pi$-action. Since the odd dimensional oriented cobordism groups of smooth free $\pi$-manifolds are torsion [CF] for finite $\pi$, a finite multiple $s \tilde{M}$ of $\tilde{M}$ bounds a $\pi$-manifold $Q$. We take the same multiple of the universal cover $\tilde{W}$ of $W$ and attach $Q$ on both ends. Let $V$ be the resulting closed manifold. Then

$$
\sigma(V)=\sigma(Q)+\sigma(s \tilde{W})+\sigma(-Q)=s \sigma(\tilde{W}) .
$$

By the equivariant Atiyah-Singer index theorem [AS] (6.2) the $\pi$-signature of a free closed $\pi$-manifold is a multiple of the regular representation, i.e., it vanishes on elements $g \neq 1$ of $\pi$ if considered as a character of the group $\pi$. Thus $\chi(l)$ is a multiple of the regular representation and therefore up to finite torsion

$$
l \in L_{n+1}(1) \in L_{n+1}(\pi) .
$$

On the other hand the subgroup $L_{n+1}(1)$ of $L_{n+1}(\pi)$ acts on $\mathscr{S}(\mathscr{M})$ with finite orbit. This can be seen from the diagram:

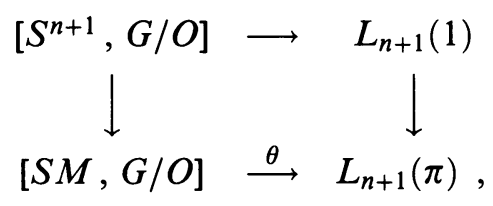

and the fact that the upper horizontal map is a rational isomorphism by the classification of homotopy spheres. This implies that the subgroup

$$
\theta([S M, G / O]) \cap L_{n+1}(1) \subseteq L_{n+1}(\pi)
$$

has finite index in $L_{n+1}(1)$. Since by exactness $\theta([S M, G / O]) \cap L_{n+1}(1)$ operates trivially on $\mathscr{S}(M), L_{n+1}(1)$ operates with finite orbit. This completes the proof of the proposition.

Proof of Theorem 4. The arithmeticity of the group $\operatorname{aut}_{t}^{s}(M)$ of simple selfhomotopy equivalences which preserve the tangent bundle follows from the sequence of inclusions

$$
\operatorname{diff}(M) \subseteq \operatorname{aut}_{t}^{s}(M) \subseteq \operatorname{aut}_{t}(M)
$$

and Proposition 15.

\section{REFERENCES}

[AS] M. Atiyah and I. Singer, The index of elliptic operators III, Ann. of Math. 87 (1968), 5460-604.

[B] A. Borel, Linear algebraic groups, Benjamin, New York, 1969. 
[BH] A. Borel and Harish-Chandra, Arithmetic subgroups of algebraic groups, Ann. of Math 75 (1962), 485-535.

[BS] A. Borel and J. P. Serre, Corners and arithmetic groups, Comment. Math. Helv. 48 (1973), 436-491.

[BSz] E. Brown and R. Szczarba, Rational and real homotopy theory with arbitrary fundamental groups, preprint, 1990.

[CF] P. Conner and E. Floyd, Differential periodic maps, Springer-Verlag,, Heidelberg, New York, 1964.

[DDK] E. Dror, W. Dwyer and D. Kan, Self homotopy equivalences of virtually nilpotent spaces, Comment. Math. Helv. 56 (1981), 599-614.

[H] S. Halperin, Lectures on minimal models, Mem. Soc. Math. France 9 (1983).

[Hu] J. Humphreys, Linear algebraic groups, Graduate Texts in Math., vol. 21, Springer-Verlag, 1975.

[P] T. Petrie, The Atiyah-Singer invariant, the Wall groups $L(\pi, 1)$, and the function $\left(t^{x}+1\right)$ / $\left(t e^{x}-1\right)$, Ann. of Math. 92 (1970), 174-187.

[S] D. Sullivan, Infinitesimal computations in topology, Publ. Math. IHES 47 (1978), 269-331.

[T1] G. Triantafillou, Aequivariante Rationale Homotopietheorie, Bonner Math. Schriften 110, Bonn, 1978.

[T2] _ An algebraic model for G-homotopy types, Astérisque 113-114 (1984), 312-337.

[T3] , Diffeomorphisms of manifolds with finite fundamental group, Bull. Amer. Math. Soc. 31 (1994), 50-53.

[W] C. T. C. Wall, Surgery on compact manifolds, Academic Press, London New York, 1970.

[Wi] C. Wilkerson, Minimal simplicial groups, Topology 15 (1976), 111-130.

Department of Mathematics, Temple University, Philadelphia, Pennsylvania 19122 E-mail address: georgia@euclid.math.temple.edu 